\title{
Magnetic Resonance Imaging Findings in Spondylodiscitis
}

\author{
Om Biju Panta, ${ }^{1}$ Yagya Raj Pathak, ${ }^{1}$ Dan Bahadur Karki² \\ ${ }^{1}$ Department of Radiology and Imaging, Koshi Zonal Hospital, Biratnagar, Nepal, ${ }^{2}$ Department of Radiology and \\ Imaging, Patan Academy of Health Sciences (PAHS), Lagenkhel, Lalitpur, Nepal.
}

\begin{abstract}
Background: Magnetic Resonance Imaging is the imaging modality of choice for imaging spinal infection due to its high sensitivity and specificity. This study aims to study the magnetic resonance imaging changes in patients with spondylodiscitis.

Methods: The study was a retrospective study carried in a multimodality imaging centre in Kathmandu. Magnetic resonance imaging records and clinical record of 3 years duration were reviewed and patients with clinical and radiological diagnosis of spondylodiscitis were included in the study. Three radiologists interpreted Magnetic Resonance Imaging with mutual consensus in disputed issues. Data analysis was done with Statistical Package for Social Sciences21.0.

Results: A total of 52 patients were included in the study. The mean age of the patients was $43.9 \pm 17.6$ years. Spondylodiscitis involved lumbar spine in $26(50 \%)$ case, cervical and thoracic spine in $13(25 \%)$ cases each. Multiple IV discs were involved in 24(46.2\%) cases, which was most common in cervical spine and least common in thoracic spine. Only one vertebral end plate was involved in 16(30.8\%) cases. Epidural collection was seen in 23(44.2\%) cases and paravertebral collection was noted in 63(33.5\%) cases. Statistical significant difference in region of spine involved $(\mathrm{p}=0.02)$ and epidural collection $(\mathrm{p}=0.04)$ was noted between genders.

Conclusions: Lumbar spine was the most common level involved with spondylodiscitis, perivertebral enhancing soft tissue was present in all cases, and involvement of disc and the endplates were the most common pattern.

Keywords: Epidural collection; magnetic resonance imaging; modic changes; spondylodiscitis.
\end{abstract}

\section{INTRODUCTION}

Infection of the spine accounts for $2-4 \%$ of all skeletal infection. ${ }^{1}$ Spondylodiscitis involves intervertebral disc and its adjacent vertebral bodies but later may also extend into the perispinal region, epidural region and over several spinal segments. Also the diagnosis is delayed due to non-specific and variable clinical findings. The area has relatively restricted access to aspiration and culture and imaging guidance is required for obtaining the material. In this context, imaging remains the main stay of diagnosis, localization and follow up. ${ }^{2-3}$ Magnetic Resonance Imaging (MRI) due to its multi plananr capabilities, excellent soft tissue contrast and simultaneous visualization of neural elements is the modality of choice for imaging of spinal infection. ${ }^{4}$ However on MRI conditions like degenerative vertebral changes (Modic changes) ${ }^{5}$ spondyloarthropathies may be mistaken for spondylodiscitis. However findings like disc changes: T2 hyperintensity, loss of nuclear cleft, decreased disc height, erosion and destruction of endplates, and perivertebral and epidural soft tissue edema are more favorable to infective spondylodiscitis. ${ }^{6}$

The study of spondylodiscitis in MRI in our country, where infective pathologies and spondylodiscitis are quite common, is not abundant. This study aims to identify MRI characteristics in patients with radiological and clinical diagnosis of spondylodiscitis in Nepal and help differentiate the common degenerative changes in spine with this treatable infective pathology.

\section{METHODS}

The study was a hospital record based retrospective study conducted in Kathmandu Imaging, a multimodalityimaging center in Kathmandu associated with a tertiary care neurological center. MRI of spine (cervical, dorsal
DOI:http://dx.doi.org/10.3126/jnhrc. $\mathrm{v} 15 \mathrm{i} 3.18843$ 
or lumbar) from May 2010 to May 2013 was reviewed and all patients with diagnosis of spondylodiscitis were included in the study. All MRI were performed with a Siemens (Magnetom C) 0.35 Tesla MRI machine. MRI images of the patients were obtained by using medium and large sized body coil with read matrix of 256 . T1 and T2 weighted images were obtained in axial and sagittal plane. Short Tau Inversion Recovery (STIR) image was obtained in sagittal plane. Contrast enhanced MRI were obtained in axial, sagittal and coronal plane in some cases.

All MRIs were interpreted by three radiologists, one of them with more than 10 years experience in spine imaging. Disputes were resolved with mutual consensus among the three radiologists.

Spondylodiscitis was diagnosed on MRI findings of T2 high signal intensity of IV disc, with marrow signal change in adjacent vertebra (T1 low and T2 high) with endplate irregularity and with or without enhancing soft tissue mass or collection surrounding the adjacent vertebral level in perivertebral and epidural space. The case was clinically assessed and diagnosis of infective spondylodiscitis was confirmed by clinical and laboratory tests.

Data was entered in SPSS spread sheet and analysis was done with IBM SPSS 21.0. Difference between means was calculated by using one-way ANOVA for more than two variables and t-test for two variables. Difference in categorical variables was calculated by using chi square test.

\section{RESULTS}

A total of 52 cases met the inclusion criteria and were included in the study. The mean age of the patients with spondylodiscitis was $43.9 \pm 17.6$ years with median of 47 years and interquartile range 28.5 and minimum age of 12 years and maximum of 83 years. There were $28(53.8 \%)$ males, and 24(46.2\%) females. Spondylodiscitis involved lumbar spine in $26(50 \%)$ case, cervical and thoracic spine in $13(25 \%)$ cases each.

\begin{tabular}{|c|c|c|c|c|c|c|c|c|c|c|}
\hline & D3-D4 & D4-D5 & D5-D6 & D6-D7 & D7-D8 & D8-D9 & D9-D10 & D10-D11 & D11-D12 & D12-L1 \\
\hline$N(\%)$ & $1(1.9)$ & $2(3.8)$ & $1(1.9)$ & $4(7.7)$ & $4(7.7)$ & $2(3.8)$ & $1(1.9)$ & $2(3.8)$ & $1(1.9)$ & $4(7.7)$ \\
\hline \multicolumn{3}{|c|}{ Cervical IV disc } & \multicolumn{2}{|l|}{$\mathrm{C} 3-\mathrm{C} 4$} & \multicolumn{2}{|l|}{$\mathrm{C} 4-\mathrm{C} 5$} & $\mathrm{C} 5-\mathrm{C} 6$ & \multicolumn{2}{|l|}{$\mathrm{C} 6-\mathrm{C} 7$} & C7-D1 \\
\hline \multicolumn{3}{|c|}{ Number(\%) } & \multicolumn{2}{|l|}{$3(5.8)$} & \multicolumn{2}{|l|}{$6(11.5)$} & $7(13.5)$ & \multicolumn{2}{|l|}{$5(9.6)$} & $3(5.8)$ \\
\hline \multicolumn{3}{|c|}{ Lumbar IV disc } & \multicolumn{2}{|l|}{ L1-L2 } & \multicolumn{2}{|l|}{ L2-L3 } & L3-L4 & \multicolumn{2}{|l|}{ L4-L5 } & L5-S1 \\
\hline \multicolumn{3}{|c|}{ Number(\%) } & \multicolumn{2}{|l|}{$6(11.5)$} & \multicolumn{2}{|l|}{$3(5.8)$} & $3(5.8)$ & \multicolumn{2}{|l|}{$5(9.6)$} & $9(17.3)$ \\
\hline
\end{tabular}

There was involvement of multiple IV disc in 24(46.2\%) cases and only one IV disc was involved in 28(53.8\%) cases. Multiple IV disc involved was most common in cervical spine involving $9(17.3 \%)$ cases followed by lumbar $8(15.4 \%)$ cases and thoracic in $7(13.5 \%)$ cases. The most common IV disc level involved was L5-S1 (9; $17.3 \%)$ followed by C5-C6 $(7 ; 13.5 \%)$ cases. (Table 1. , Table 2., Table 3.).

Involvement of vertebra was noted in form of endplate irregularity and marrow signal change. Involvement of multiple vertebrae (at least two level) was noted in $36(69.2 \%)$ cases and single vertebra involvement was noted in $16(30.8 \%)$ cases. Only one vertebra involvement is seen early in course of disease. Only one vertebra endplate involvement was noted most frequently in lumbar spine $(14 ; 26.9 \%)$ followed by cervical spine $(2$; $3.8 \%)$.

Epidural collection was noted in 23(44.2\%) cases, most frequently seen at dorsal spine followed by lumbar spine and rare at cervical spine. (Table 4.) However, significant compression of spinal cord was noted in only two cases in dorsal spine and none in lumbar and cervical spine. Paravertebral collection was noted in 33(63.5\%) of all cases. All cases of dorsal spine spondylodiscitis demonstrated either prevertebral or paravertebral collection. Perivertebral collection was lest common in cervical spine spondylodiscitis with only $4(30.8 \%)$ cases demonstrating either a prevertebral or paravertebral collection (Table 4). Minimal perivertebral T2 high signal intensity soft tissue was however noted in all cases. There were no cases with involvement of posterior elements in our study. In 3(5.8) cases of thoracic spondylodiscitis, prevertebral collection tracking under anterior longitudinal ligament with scalloping of the anterior vertebral body was noted.

There was no significant variation among gender in involvement of the IV disc, vertebral endplates and perivertebral collection. However, significant difference among gender in involvement of the spine region and presence of epidural component was noted (Table 4). 


\begin{tabular}{|c|c|c|c|c|}
\hline & $\begin{array}{r}\text { Cervical } \\
N=13\end{array}$ & $\begin{array}{r}\text { Dorsal } \\
N=13\end{array}$ & $\begin{array}{r}\text { Lumbar } \\
N=26\end{array}$ & $\begin{array}{r}\mathrm{p}- \\
\text { value }\end{array}$ \\
\hline Mean Age & 39.616 .1 & $\begin{array}{l}45.5 \\
14.1\end{array}$ & 45.319 .8 & $0.604^{*}$ \\
\hline \multicolumn{5}{|l|}{ Sex } \\
\hline $\begin{array}{l}\text { Male } \\
\text { Female }\end{array}$ & $\begin{array}{l}4(30.8) \\
9(69.2)\end{array}$ & $\begin{array}{r}11(84.6) \\
2(15.4)\end{array}$ & $\begin{array}{l}13(50.0) \\
13(50.0)\end{array}$ & $0.02^{* *}$ \\
\hline \multicolumn{5}{|c|}{ Disc Involved } \\
\hline $\begin{array}{l}\text { Single } \\
\text { Multiple }\end{array}$ & $\begin{array}{l}4(30.8) \\
9(69.2)\end{array}$ & $\begin{array}{l}6(46.2) \\
7(53.8)\end{array}$ & $\begin{array}{r}18(69.2) \\
8(30.8)\end{array}$ & $0.06^{* *}$ \\
\hline
\end{tabular}

Vertebra (endplate) involved

\begin{tabular}{lrrrr} 
Single & $2(15.4)$ & 0 & $14(53.8)$ & \\
Multiple & $11(84.6)$ & $13(100)$ & $12(46.2)$ & $0.01^{* *}$ \\
$\begin{array}{l}\text { Epidural } \\
\text { collection }\end{array}$ & $3(23.1)$ & $7(53.8)$ & $13(50 \%)$ & 0.2 \\
$\begin{array}{l}\text { Peri- } \\
\text { vertebral } \\
\text { collection }\end{array}$ & $4(30.8)$ & $13(100)$ & $16(61.5)$ & $0.001^{* *}$ \\
\hline
\end{tabular}

* Calculated by one-way ANOVA. ** Calculated by Chi Square Test. Bold Statistically significant

Table 4. Variation in MRI findings in spondylodiscitis according to gender.

$\begin{array}{rrr}\text { Male } & \text { Female } & \begin{array}{c}\mathrm{p}- \\ \mathrm{N}=28\end{array} \\ \mathrm{~N}=24 & \text { value }\end{array}$

Region of spine involved

\begin{tabular}{lrrr} 
Cervical & $4(14.28)$ & $9(37.5)$ & \\
Dorsal & $11(39.28)$ & $2(8.3)$ & 0.02 \\
Lumbar & $13(46.4)$ & $13(54.2)$ & \\
$\begin{array}{l}\text { Disc Involved } \\
\text { Single }\end{array}$ & & \\
Multiple & $14(50.0)$ & $14(58.3)$ & 0.55 \\
Vertebra (endplate) & involved & & \\
Single & $6(21.4)$ & $10(41.7)$ & 0.11 \\
Multiple & $22(78.6)$ & $14(58.4)$ & \\
Epidural collection & $16(57.1)$ & $7(29.2)$ & 0.04 \\
$\begin{array}{l}\text { Peri-vertebral } \\
\text { collection }\end{array}$ & $20(71.4)$ & $13(54.2)$ & 0.19 \\
\hline
\end{tabular}

\section{DISCUSSION}

MRI is the modality of choice for spine imaging due to its high sensitivity to soft tissue and marrow edema and also its ability resolve neural elements as separate from spinal column. ${ }^{2}$ Anatomically the most common region involved in spondylodiscits is lumbar spine and least common involved region is cervical spine. ${ }^{2-7}$ In our study as well lumbar spine was the most common region involved accounting for $50 \%$ of cases and thoracic and cervical spines were each involved in $25 \%$ of cases. The mean age of the patients in our study was $43.9 \pm$ 17.6 years slightly lower than reported in previous studies which has reported mean age of 59 years. ${ }^{8}$ The difference might represent the regional variation in epidemiology of infection as previous studies have been conducted in west where infections are usually limited to immunosuppressed elderly populations. Spondylodiscitis is reported to have a bimodal distribution with peak at less than 20 years and second peak at 50-70 years. ${ }^{4}$ There was only slight male predominance in our study; while a definite male predisposition with male to female ratio of 1.5-2:1 has been previously reported. ${ }^{4,8}$

Infection of the spine may involve vertebral body (spondylitis), intervertebral disc (discitis), both disc and vertebral body (spondylodiscitis), perivertebral soft tissue (pre and paravertebral abscess) and epidural tissue (Epidural abscess), meninges and arachnoid and rarely spinal cord (myelitis, abscess). ${ }^{7}$ All cases in our series had spondylodiscitis with some having epidural and paravertebral abscess as well. There was involvement of multiple IV disc in $46 \%$ cases in our study. Pyogenic spondylodiscitis usually involves single vertebral segment that is one intervertebral disc and adjacent two vertebral bodies whereas involvement of more than 3 vertebral levels is usually seen in tubercular spondylodiscitis. $^{3}$ In our study, almost half of the population had involvement of two or more IV disc i.e. multiple vertebral segment involvement, which probably might be due to tubercular spondylodiscitis and points towards high prevalence of tuberculosis in our part of the world. However involvement of multiple vertebral levels has also been described in non-tubercular infections as well.9-10 Differentiation between pyogenic and tubercular spondylodiscitis has been attempted on MRI based on many characteristics, however MRI does not have adequate sensitivity and specificity to differentiate the two condition and one most rely on invasive tissue sampling and diagnosis. ${ }^{11}$ Bacteriological diagnoses were not analyzed in our study. Also we identified involvement of single intervertebral disc and only single vertebral endplate in $30 \%$ of cases. The typical finding of spondylodiscitis on MRI is involvement of both adjacent vertebral endplate on either side of intervertebral disc $\mathrm{C}^{1,2,7}$ However, early in course of disease there may be involvement of only one endplate, which later progresses to involve both the endplates. ${ }^{10-12}$ 
The prevalence of single intervertebral disc involvement was quite high in our study as compared to previous studies. ${ }^{10,13}$ Isolated infection of vertebral body known, as spondylitis was not seen in our study.

Paraspinal or epidural inflammatory tissue has been described to be invariably associated with spinal infection and is also a criteria used diagnose spondylodiscitis. ${ }^{10,14}$ In our study as well, all cases had some amount of inflammatory tissue in perivertebral and epidural region as T2 high signal intensity soft tissue. Para spinal and epidural collection was identified on MRI by fluid signal intensity area in paraspinal or epidural region, which showed rim enhancement on contrast MRI. Paravertebral and epidural collection was seen in almost half of the patients in our study. However, only few of them were large enough and approachable for percutaneous drainage. Large collection with welldefined smooth wall is seen in tuberculosis, where as in pyogenic spondylodiscitis phelgmon or abscess is illdefined with irregular wall and demonstrates irregular rim or diffuse enhancement. ${ }^{3,8,15}$ Distinction between phelgmon and abscess is important as phlegmon can be treated conservatively with antibiotics while abscess requires drainage. ${ }^{8}$ There was variation among gender noted in presence of epidural collection and involvement of spine region, however we consider the difference to be a matter of chance than an actual difference. A study with a larger sample size and study of other risk factors as well might be required to clarify the doubt.

There were certain limitations of our study. Only patients undergoing MRI were selected for the study which may not be true representation of patients with spondylodiscitis Bacteriological diagnosis was not analyzed in our study. Observer variability in reporting the MRI could lead to potential bias with identifying findings and diagnosing spondylodiscitis.

\section{CONCLUSIONS}

In conclusion, spondylodiscitis most frequently involved the lumbar spine, males were more frequently involved than females. Involvement of IV disc and both endplates though a common finding in spondylodiscitis involvement of single vertebra along with IV disc was also relatively common. Perivertebral enhancing soft tissue was universal in all patients with spondylodiscitis and can be a reliable sign to differentiate degenerative vertebral changes (Modic changes) from spondylodiscitis.

\section{REFERENCES}

1. Maiuri F, Iaconetta G, Gallicchio B, Manto A, Briganti F. Spondylodiscitis. Clinical and magnetic resonance diagnosis. Spine. 1997;22(15):1741-6. [Link]

2. Diehn FE. Imaging of Spine Infection. Radiol Clin North Am. 2012;50(4):777-98. [ScienceDirect]

3. Sung Hwan H, Ja-Young C, Joon Woo L, Na Ra K, JungAh C, Heung Sik K. MR Imaging Assessment of the Spine: Infection or an Imitation? RadioGraphics. 2009;29(2):599612. [Link]

4. Gouliouris T, Aliyu SH, Brown NM. Spondylodiscitis: update on diagnosis and management. J Antimicrob Chemother. 2010;65(suppl 3):iii11-iii24. [FullText]

5. Modic MT, Masaryk TJ, Ross JS, Carter JR. Imaging of degenerative disk disease. Radiology. 1988;168(1):17786. [Link]

6. Stabler A, Reiser MF. Imaging of spinal infection. Radiol Clin North Am. 2001;39(1):115-35. [Link]

7. Sharif HS. Role of MR imaging in the management of spinal infections. Am J Roentgenol. 1992;158(6):133345. [Link]

8. Sans N, Faruch M, Lapègue F, PonsotA, Chiavassa H, Railhac JJ. Infections of the spinal column - Spondylodiscitis. Diagn Interv Imaging. 2012;93(6):520-9. [ScienceDirect]

9. Modic MT, Feiglin DH, Piraino DW, Boumphrey F, Weinstein MA, Duchesneau PM et al. Vertebral osteomyelitis: assessment using MR. Radiology. 1985;157(1):157-66. [Link]

10. Ledermann HP, Schweitzer ME, Morrison WB, Carrino JA. MR imaging findings in spinal infections: rules or myths? Radiology. 2003;228(2):506-14. [Link]

11. Frel M, Białecki J, Wieczorek J, Paluch , DąbrowskaThing A, Walecki J. Magnetic Resonance Imaging in Differentatial Diagnosis of Pyogenic Spondylodiscitis and Tuberculous Spondylodiscitis. Pol J Radiol. 2017;82:7187. [Link]

12. Shih TT, Huang KM, Hou SM. Early diagnosis of single segment vertebral osteomyelitis--MR pattern and its characteristics. Clin Imaging. 1999;23(3):159-67. [ScienceDirect]

13. Ziegelbein j, el-khoury gy. Early spondylodiscitis presenting with single vertebral body involvement: a report of two 
cases. Iowa Orthop J. 2011;31:219-24. [FullText]

14. Thrush A, Enzmann D. MR imaging of infectious spondylitis. Am J Neuroradiol. 1990;11(6):1171-80.

15. Moorthy S, Prabhu NK. Spectrum of MR imaging findings in spinal tuberculosis. Am J Roentgen. 2002;179(4):97983. [FullText] 